RUBÉN JACOB-DAZAROLA

DEPARTAMENTO DE DISEÑO

FACULTAD DE ARQUITECTURA Y URBANISMO

UNIVERSIDAD DE CHILE

RUBENHJD@UCHILEFAU.CL

\title{
Desde la utilidad a la emoción
}

\author{
From the utility to the emotion
}

Las emociones forman parte de la vida cotidiana desde los primeros albores del ser humano y muy probablemente antes de la conciencia y la razón. Nos han permitido comprender y empatizar con los demás, facilitando la socialización y las relaciones entre los individuos al actuar como mecanismo de intercambio de información sobre el sentir de los otros.

Estas emociones pueden ser definidas de muy diversa manera dependiendo de la perspectiva con que sean analizadas. Una de estas definiciones plantea que las emociones corresponden a respuestas psicofisiológicas ante estímulos ambientales, que desencadenan estos procesos emocionales permitiendo a los individuos una reacción coherente con la naturaleza peligrosa o benigna del estímulo. No obstante, las emociones humanas se establecen como algo mucho más complejo y que se entremezcla con la infinidad de aspectos que implica formar parte de nuestra especie.

A fines del siglo XIX, a partir del trabajo de Darwin (1872) y de James (1884) surgen los dos primeros enfoques que intentan estudiar y explicar estos procesos de modo sistemático, desde una perspectiva evolutiva el primero, donde las emociones corresponden a adaptaciones que facilitan la sobrevivencia, y a partir de las respuestas corporales asociadas a las diversas emociones el segundo. La más reciente aparición de perspectivas de carácter híbrido, entre las cuales cabe destacar el enfoque cognitivo, propone una integración entre los determinantes fisiológicos, sociales y cognitivos de las emociones (Frijda, 1988; Schachter, y Singer, 1962), como una explicación de mirada más amplia a este fenómeno.

La interacción del ser humano con su entorno ha cambiado mucho desde la aparición de los primeros hombres, y ese carácter utilitario que se le asignaba a las emociones, al ser respuestas que permitían reacciones pertinentes frente a un estímulo determinado, no está siempre presente. Para cualquiera resultará familiar la idea de un espectador emocionándose hasta las lágrimas al ver una película o frente a una pieza musical de su preferencia. Así entonces, las emociones humanas no obedecen solo a códigos instrumentales, sino también estéticos, sociales y culturales (Desmet, 2003). Es esta característica de ser desencadenadas no solamente por estímulos urgentes, sino también por la contemplación, la interacción y la apropiación del entorno mediante los sentidos, el punto de encuentro preliminar entre el diseño y las emociones, entre aquellas cosas creadas por el hombre que son capaces de provocar reacciones emocionales en las personas que las utilizan.

Durante el año 1999 tuvo lugar en la ciudad de Delft, Holanda, la primera conferencia internacional de Diseño y Emociones, mismo año en que se creó la International Design and Emotion Society. Estos dos hitos fueron claves en el establecimiento formal de un enfoque académico sobre esta temática que se expandió en diferentes áreas de investigación y que hoy en día da lugar a los más recientes planteamientos en este ámbito, tales como el Diseño positivo, o el Diseño para el bienestar y la felicidad (Desmet, 2013; Desmet \& Pohlmeyer, 2013; Jiménez, Pohlmeyer y Desmet, 2016).

Además, durante el desarrollo del enfoque emocional del Diseño se han tratado, y se continúan tratando, temáticas tales como las propiedades expresivo-sen- 
soriales de los materiales (Karana, Pedgley \& Rognoli, 2014), las relaciones que se establecen entre las personas y los objetos (Mugge, Schifferstein \& Schoormans, 2006), así como las personalidades que poseen estos últimos (Desmet, Ortiz N. \& Schoormans, 2008), el aprovechamiento de las emociones negativas en el desarrollo de nuevos productos (Fokkinga, Desmet \& Hoonhout, 2010), la experiencia multimodal que se vive al utilizar un producto (Ortiz N., y Aurisicchio, 2011; Schifferstein \& Hekkert, 2008), entre muchas otras, dando cuenta de una mirada amplia, profunda, y también capaz de renovarse a sí misma.

Si el trabajo de Donald Norman y su libro El Diseño emocional (Norman, 2004) resultó clave para que esta manera de aproximarse al Diseño se diera a conocer masivamente, permeando así a los futuros usuarios, fabricantes y a los diversos agentes que forman parte del ciclo del Diseño, la labor realizada en el contexto académico por figuras como Pieter Desmet, Paul Hekkert, Hendrick Schifferstein, Ruth Mugge o Marco van Hout entre muchos otros, resultó igualmente imprescindible para el establecimiento de las bases académicas del enfoque, contribuyendo de forma relevante a su credibilidad, permanencia en tiempo y profundidad, así como también a la propuesta constante de nuevas miradas dentro del ámbito del diseño y las emociones, que permiten permanecer optimistas sobre su desarrollo próximo y su aporte a la disciplina.

\section{El dossier temático en RChD}

En este número de RChD: Creación y Pensamiento estos y otros diferentes tópicos vinculados al enfoque emocional del Diseño son abordados de manera especial por los diferentes autores que han aportado con sus trabajos al dossier temático.

En el primer artículo, denominado "Materia Emocional, los materiales en nuestra relación emocional con los objetos" de Valentina Rognoli y Camilo Ayala (POLIMI, Italia), se aborda la dimensión emocional de los materiales que conforman los productos. Más allá de su utilidad práctica, valor económico o características físico-mecánicas los materiales están cargados de significados, representando y transmitiendo los mensajes que los diseñadores plasman en los productos que desarrollan. Los autores presentan los DIY-Materials (Do It Yourself Materials) y los materiales interactivos, conectados e inteligentes o ICS-Materials, y cómo estos son capaces de dar una nueva vuelta de tuerca a la relación persona-material-producto conectando a diseñadores y usuarios. Posteriormente, en "THR patient's emotional and physical assessment after early hospital discharge" Natalia Romero, Juan Jiménez Garcia, David Keyson y Paul Havinga (TU Delft, Holanda; ICESI University, Colombia; y University of Twente, Holanda), se adentran en los alcances que el Diseño tiene en el campo hospitalario, y documentan el desarrollo de ESTHER 1.0, una herramienta electrónica que permite a los pacientes autorreportar sus experiencias durante el primer período de la recuperación en su hogar.

La tercera entrega, "Billy Cash: Digital piggy bank for meaningful saving behaviour" a cargo de Santiago De Francisco y Mafalda Casais (U. de los Andes, Colombia y Tu Delft, Holanda, respectivamente) introduce el enfoque del Diseño para comportamientos virtuosos a través de un caso de estudio que plantea cómo es posible motivar al ahorro mediante el diseño de un producto tan tradicional como es una alcancía, y cómo la disciplina puede contribuir a promover actitudes deseables en las personas, dando pie a una profunda y necesaria discusión sobre los alcances ético-morales de la actividad del diseño y su rol en la sociedad. 
Con una perspectiva que conecta en variados niveles con el concepto de los comportamientos virtuosos planteados en el artículo previo, Mitzi Vielma (U. de Chile), autora del cuarto artículo denominado "La obesidad en mujeres: estrategia de diseño de productos para el bienestar físico, basados en el placer sexual", plantea una revisión en las estrategias de diseño de productos para la salud, proponiendo el placer físico obtenido en su utilización como una manera de estimular el uso continuo de productos para ejercitarse y luchar contra la obesidad.

Alexis Brantes, en "Interacción, afecto y sociedad; reflexiones desde la neurociencia afectiva y Customer Experience" aborda el análisis, desde una perspectiva cercana a la neurociencia; la relación entre las emociones y las interacciones entre las personas y los objetos; $y$ los procesos internos $y$ externos (sociales) que estas situaciones desencadenan.

En el artículo siguiente, "El factor emocional del prototipado de diseño de servicios en el laboratorio sINCO (Service Innovation Corner)", Mariluz Soto establece las relaciones existentes entre los procesos emocionales y las experiencias diseñadas, desde el enfoque conocido como ux o Diseño para la experiencia del usuario. Cada vez más los diseñadores son capaces de proponer no solamente objetos materiales, sino también servicios, sistemas e interacciones, creando situaciones vivenciales pre-diseñadas que permiten a los usuarios obtener una experiencia también previsible y por ende asociada a procesos emocionales en común.

El dossier temático finaliza con el trabajo que nos presenta Juan Carlos Ortiz Nicolás denominado "Analizando respuestas verbales para identificar evaluaciones temáticas en la interacción persona-objeto". En este artículo el Dr.

Ortiz, se refiere a los juicios que las personas realizan de forma inconsciente e inmediata cuando interactúan con productos. Estas evaluaciones, que dan posteriormente paso a las emociones, son propuestas como un punto inicial para el desarrollo de productos que puedan provocar emociones deseadas por los diseñadores y los usuarios. 


\section{Referencias}

Darwin, C. (1872). The expression of the emotions in man and animals. London: John Murray, Albermarle street.

Desmet, P. M. A. (2003). A Multilayered Model of Product Emotions. The Design Journal, 6(2), 4-13. https://doi. org/10.2752/146069203789355480

Desmet, P. M. A. (2013). Positive Design. Inaugural lecture. Delft, Holanda: Faculty of Industrial Design Engineering, TU Delft.

Desmet, P. M. A., Ortiz Nicolás, J. C., \& Schoormans, J. P. (2008). Product Personality in Physical Interaction. Design Studies, 29(5), 458-477.

Desmet, P. M. A., \& Pohlmeyer, A. E. (2013). Positive Design: An Introduction to Design for Subjective Well-Being. International Journal of Design, 7(3), 5-19. https://doi.org/10.1108/10878571011029028

Fokkinga, S. F., Desmet, P. M. A., \& Hoonhout, J. (2010). The Dark Side of Enjoyment. Using negative emotions to design for rich user experiences. 7th International Design et Emotion Conference Proceedings, 1-12.

Frijda, N. H. (1988). The Laws of Emotion. American Psychologist, 43(5), 349-358. https://doi.org/10.5465/ AMR.2007.25275690

James, W. (1884). What is an Emotion? Mind, 9(34), 188205. https://doi.org/10.1093/mind/LI.202.200
Jiménez, S., Pohlmeyer, A. E., \& Desmet, P. M. A. (2016). Diseño Positivo. Guía de Referencia. Bogotá: Universidad de los Andes.

Karana, E., Pedgley, O., \& Rognoli, V. (2014). Materials Experience. Materials Experience. Oxford, UK: Elsevier. https://doi.org/10.1016/B978-0-08-099359-1.00004-7

Mugge, R., Schifferstein, H. N. J., \& Schoormans, J. P. L. (2006). Product Attachment and Product Lifetime: The Role of Personality Congruity and Fashion. European Advances in Consumer Research, 7, 460-467.

Norman, D. A. (2004). El Diseño Emocional. Por qué nos gustan (o no) los objetos cotidianos. Barcelona: Paidós. Ortiz Nicolás, J. C., \& Aurisicchio, M. (2011). A Scenario of User Experience. In International Conference on Engineering Design Proceedings ICED11 (pp. 1-12). Denmark: Kobenhavn.

Schachter, S., \& Singer, J. E. (1962). Cognitive, Social, and Physiological Determinants of Emotional State. American Psychologist, 69(5), 379-399. https://doi. org/10.1037/hoo21465

Schifferstein, H. N. J., \& Hekkert, P. (Eds.) (2008). Product Experience. Amsterdam, Holanda: Elsevier. 